\title{
Gouverner la consommation d'énergie des ménages. Renouvellement des enjeux et des instruments d'intervention (1973-2017)
}

家庭用エネルギー消費の抑制—争点と政策の变遷（1973-2017）— Governing Households' Energy Consumption. Renewal of Issues and Policy Tools (1973-2017)

\section{Benoit Granier}

\section{(2) OpenEdition}

1 Journals

\section{Édition électronique}

URL : http://journals.openedition.org/ebisu/4560

DOI : $10.4000 /$ ebisu. 4560

ISSN : 2189-1893

Éditeur:

Institut français de recherche sur le Japon (UMIFRE 19 MAEE-CNRS), Maison franco-japonaise

\section{Édition imprimée}

Date de publication : 23 janvier 2019

Pagination : 223-252

ISSN : $1340-3656$

\section{Référence électronique}

Benoit Granier, « Gouverner la consommation d'énergie des ménages. Renouvellement des enjeux et des instruments d'intervention (1973-2017) », Ebisu [En ligne], 56 | 2019, mis en ligne le 24 décembre 2019, consulté le 17 janvier 2020. URL : http://journals.openedition.org/ebisu/4560 ; DOI : 10.4000/ ebisu. 4560 


\section{Gouverner la consommation d'énergie des ménages}

Renouvellement des enjeux et des instruments d'intervention (1973-2017)

Benoit Granier

家庭用エネルギー消費の抑制

一争点と政策の変遷（1973-2017）-

ブク・ガルニエ

Governing Households' Energy Consumption.

Renewal of Issues and Policy Tools (1973-2017)

Benoit Granier

Mots-clés : action publique, politique énergétique, changement climatique, gouvernance des conduites, shöene

L'auteur : Benoit Granier est docteur de l'université de Lyon et qualifié en sections CNU 04 (science politique) et 15 (langue et civilisation japonaises). Ses recherches en sociologie de l'action publique portent sur la transformation des politiques publiques dans les domaines de l'énergie, de l'environnement et de l'alimentation, en France et au Japon particulièrement.

Résumé : Cet article est consacréà l'évolution des enjeux et des pratiques en matière de gouvernement de la consommation d'énergie des ménages japonais depuis les chocs pétroliers des années 1970. Il met ainsi en exergue l'impact des conjonctures nationale et internationale, du progrès scientifique et technique mais aussi de l'expression des intérêts des acteurs industriels. Durant la décennie 1990, les accords internationaux sur le climat ont mis l'accent sur la promotion des comportements économes en énergie et provoqué l'entrée en scène d'un nouvel acteur: le ministère de l'Environnement. L'intervention étatique s'est à nouveau renforcée suite à l'accident de Fukushima et au développement d'instruments novateurs originaires des États-Unis, tout en restant en accord avec les objectifs de préserver la compétitivité des firmes nationales et de stimuler la croissance économique. 
キーワード

公共政策、エネルギー政策、気候変動、行動 変容、省エネ

\section{著者}

ブノワ・ガルニエ：リヨン大学で政治学及び 日本語・日本文化学の博士号を取得。研究分 野は、特にフランスと日本におけるエネル ギー、環境及び食糧政策の変遷である。

\section{要旨}

本論文では、日本の家庭用エネルギー消費の抑

Veywords: Public Policy, Energy Policy, Climate Change, Behaviour Change, Shöene

The Author: Benoit Granier obtained his $\mathrm{PhD}$ from Lyon University and is qualified by the CNU (National Commision of University Accreditation) to teach in political science and Japanese studies. His research in the sociology of public action focuses on the transformation of public policy in the fields of energy, environment and food, especially in France and Japan.

Abstract: This article examines the evolution of issues and practices relating to the governing of household
制に関して 1970 年代のオイルショック以来採 られてきた政策と争点の変遷について論じる。 本論文は国内及び国際情勢、科学やテクノ口 ジーの発展だけでなく産業界の利害が与えたイ ンパクトを強調。1990 年代は気候に関する国 際的合意により省エネルギー行動が大きく促進 され、これにより環境省が新たな利害関係者に なった。日本企業の競争力を保ち経済発展を促 進するという目的を持ちながらも、福島原発事 故やアメリカの政策の変化により、再び国の介 入が強くなっている。

energy consumption in Japan since the oil crises of the 1970s. It highlights the impact of domestic and international circumstances, scientific and technical progress, and the expression of industrial stakeholders' interests. During the 1990 s, international agreements on climate change mitigation led to increased promotion of energy-saving behaviours, establishing the Ministry of the Environment as a new stakeholder in Japan's energy policy. State intervention has increased again since the Fukushima disaster and the development of new policy tools in the United States, while retaining the objective of maintaining the competitiveness of Japanese companies and promoting economic growth. 


\section{Gouverner la consommation d'énergie des ménages}

Renouvellement des enjeux et des instruments d'intervention (1973-2017)

Benoit Granier*

L'État japonais présente une longue histoire d'interventions dans la vie quotidienne des citoyens : il a ainsi mis en œuvre de nombreux programmes visant à influencer leur conduite. Certains domaines d'action publique ont fait l'objet de nombreuses études, à l'image de la police (Westney 1982), des politiques sanitaires et sociales (Thomann 2015), de la gestion des populations ouvrières (Thomann 2010) ou des finances des ménages (Garon 1997). Le gouvernement des conduites ${ }^{1}$ (Dubuisson-Quellier 2016) dans le domaine de la consommation d'énergie reste toutefois moins examiné par les chercheurs japonais et occidentaux car l'intérêt des ministères concernés pour les comportements individuels est un peu plus récent. En effet, si l'État japonais a commencé à prêter attention aux pratiques de consommation énergétique de ses citoyens depuis les années 1970, ses interventions se sont surtout intensifiées à partir de la fin des années $2000^{2}$.

1. Défini par Sophie Dubuisson-Quellier comme le processus par lequel « l'État intervient dans de nombreux champs d'action publique pour orienter les comportements individuels au nom de l'intérêt collectif " (Dubuisson-Quellier 2016 : 15).

2. Même si l'on peut considérer que les campagnes de promotion de la frugalité menées à partir des années 1920 ont touché par ricochet les pratiques de consommation d'énergie des ménages (Garon $1997:$ 8-10).

* Chercheur associé à l'Institut d'Asie Orientale (UMR 5062). 
La politique énergétique japonaise a longtemps été, principalement, une politique de l'offre, dont l'objectif était d'augmenter les capacités de production afin d'alimenter une demande en progression constante tout au long de la période de Haute croissance (Yamaji 2015). Toutefois, les chocs pétroliers de 1973 puis de 1979, suivis d'une mise à l'agenda national et mondial de la lutte contre le changement climatique dans les années 1990, ont changé la donne.

Le ministère du Commerce international et de l'Industrie (Tsūshō sangyōshō 通商産業省, MITI [Ministry of International Trade and Industry]), rebaptisé ministère de l'Économie, du Commerce et de l'Industrie (Keizai sangyōshō 経済産業省, METI [Ministry of Economy, Trade and Industry]) en 2001, puis l'agence de l'Environnement (Kankyōchō 環境庁), promue ministère de l'Environnement (Kankyōshō 環境省, MEO [Ministry of the Environment]) en 2001, sont ainsi devenus des acteurs majeurs du gouvernement des comportements de consommation. Ils ont contribué au premier chef à mettre en place, sur un laps de temps relativement court, de nombreux dispositifs dont le but explicite est de modifier l'utilisation par les ménages de différentes sources d'énergie ${ }^{3}$. Depuis une dizaine d'années en effet, la promotion des shöene ködōo 省エネ行動 (comportements efficients ou économes en énergie ${ }^{4}$ ) s'est imposée comme un enjeu prioritaire pour ces deux ministères, dans le cadre de leurs objectifs de mâ̂trise de la demande en énergie (shōenerugī 省エネルギー ou shōene 省エネ) issue du secteur résidentiel, c'est-à-dire des ménages ${ }^{5}$. Or cette dimension

3. Nous écartons le ministère du Territoire, des Infrastructures, des Transports et du Tourisme (Kokudo kōtsushō 国土交通省, MLIT [Ministry of Land, Infrastructure, Transport and Tourism]) dans la mesure où cet article se concentre sur l'enjeu de la consommation d'énergie, même si cela touche au secteur résidentiel.

4. Notons que le mot shōene convoque les notions voisines mais distinctes d'efficacité énergétique et d'économies d'énergie : la première se réfère à la performance des appareils électriques, sans que des économies d'énergie soient systématiques - comme en cas d'usage accru des appareils. Inversement, on peut réaliser des économies d'énergie sans améliorer l'efficacité des appareils, en réduisant par exemple l'usage du climatiseur. En tant que slogan, le mot shōene possède donc une polysémie qui le rend difficilement traduisible. C'est pourquoi nous l'employons tel quel, en particulier lorsque nous analysons le contenu des politiques et des stratégies concernées.

5. Également nées dans le contexte des chocs pétroliers des années 1970, les politiques cherchant à modifier les comportements dans le domaine de l'énergie se sont multipliées 
de la politique énergétique japonaise a jusqu’à présent été peu étudiée pour plusieurs raisons. En premier lieu, elle adopte une approche techno-économique qui accorde historiquement peu d'importance à la question des comportements (Nakagami 2010). Ensuite, les recherches ont privilégié d'autres aspects de la politique énergétique, comme ses enjeux économiques et technologiques (Leprêtre 2016; Scalise 2013; Fukasaku 2001), son articulation avec la politique climatique (Yamaji 2015; Watanabe 2011; Oshitani 2006) ou son rapport à l'énergie nucléaire, surtout depuis mars 2011 (Lévy \& Ribault 2012).

C'est pourquoi cet article propose de retracer l'évolution des dispositifs instaurés par le METI et le MOE dans l'optique de réduire la demande en énergie par la modification du comportement des ménages. Il montre que l'intervention étatique dans la vie quotidienne s'est approfondie sous l'effet d'une combinaison hétérogène d'événements et de processus à la fois internationaux et nationaux. De plus, la sophistication des instruments mis en œuvre nous renseigne plus généralement sur les transformations de l'action publique japonaise, notamment lorsqu'il est question de gouvernement des conduites. Il apparaît ainsi que les chocs pétroliers, l'enjeu du changement climatique et l'accident de Fukushima n'expliquent qu'en partie le caractère plus strict des mesures prises en matière de changement des comportements. Le renforcement de ces mesures résulte également du constat de l'inefficacité des politiques antérieures, du développement de nouvelles techniques d'intervention suite au progrès des technologies de l'information et de la communication ainsi que des connaissances sur les comportements, et paradoxalement autant des intérêts des acteurs industriels et du METI que de ceux du MOE.

Cet article s'appuie sur diverses sources collectées entre 2013 et 2017 au cours de notre recherche doctorale (Granier 2017). La couverture de la période allant des chocs pétroliers jusqu’à la fin des années 1990 repose sur une revue de la littérature existante (en anglais et en japonais) et sur des entretiens avec des hauts fonctionnaires et des experts du METI et du MOE. Le traitement des décennies 2000 et 2010 s'appuie sur le même

au cours des années 2000 dans la plupart des pays de l'OCDE (Shove 2010). Elles ont connu des transformations comparables au cas japonais, comme nous le verrons dans la quatrième partie de cet article. 
type de matériau, auxquels s'ajoutent une analyse exhaustive de textes officiels (plans d'action, rapports, etc.) et la retranscription des discussions du Shōenerugī shōiin-kai 省エネルギー小委員会 ou Comité shōene du METI, principal pourvoyeur d'expertise ad hoc pour le ministère, sur la période 2001-2017. Au total, une quarantaine d'entretiens avec des parties prenantes et des observateurs de la politique énergétique japonaise ont été réalisés entre le printemps 2014 et l'été 2016 pour obtenir des documents de première main et recouper des informations complémentaires.

Notre démonstration se structure en quatre parties correspondant à autant de moments-clés de la politique énergétique japonaise, au cours desquels des éléments nouveaux se sont juxtaposés aux acteurs et aux instruments antérieurs du gouvernement des conduites. Nous verrons d'abord comment les chocs pétroliers ont donné naissance à la politique shōene sous la juridiction du MITI. Quoique focalisée sur le secteur industriel, cette politique inaugure les premiers dispositifs de promotion des comportements shöene ${ }^{6}$, dans la lignée des techniques de gouvernement des décennies précédentes. Puis nous expliquerons comment l'agenda climatique a suscité l'irruption d'un nouvel acteur, l'agence de l'Environnement, et donné plus d'importance aux comportements shöene en accord avec les intérêts du MITI et du secteur industriel. Dans un troisième temps, nous montrerons comment le développement des technologies de l'information et de la communication a contribué à la mise en œuvre de campagnes de communication originales, après l'entrée en vigueur du protocole de Kyoto en 2005. Enfin, notre dernière partie analysera, dans un contexte marqué par la remise en question des mesures antérieures et par l'accident de Fukushima, la mobilisation par le METI et le MOE d'instruments inédits en provenance des États-Unis, fondés sur les sciences comportementales (kōdo kagaku 行動科学, comprenant l'économie comportementale et la psychologie) et le big data. Nous défendons l'argument que l'engagement à réduire les émissions de gaz à effet de serre, en s'articulant à l'expression des intérêts du METI et des acteurs industriels, aboutit au renforcement des dispositifs de gouvernement des conduites. Cette orientation politique

6. L'emploi de l'expression shōene kōdō n'est toutefois pas encore courant à l'époque, et ne deviendra incontournable chez les acteurs de la politique énergétique qu’à partir des années 2000 . 
reporte donc vers les individus une partie des efforts qui pourraient incomber aux entreprises, tout en encourageant les ménages à acheter des appareils efficients, pour entretenir la croissance économique.

\section{La naissance de la politique shōene et les premiers dispositifs de gouvernement des conduites}

Les préoccupations de l'État japonais pour l'énergie se sont longtemps résumées à l'augmentation de la production nationale. Jusqu'au début des années 1970 en effet, le principal enjeu de la politique énergétique est d'accroître l'offre pour répondre à une demande en constante progression (Yamaji 2015 : 58-61). Mais les chocs pétroliers de 1973 et 1979 bouleversent la stratégie japonaise : après la récession de 1974, le principal défi est de garantir la sécurité énergétique, c'est-à-dire d'assurer la stabilité de l'approvisionnement en énergie (Yamaji 2015: 61-62). Afin de réduire la dépendance de l'archipel vis-à-vis des pays du Golfe persique, priorité est donnée à la diversification des importations de matières premières, au développement des énergies renouvelables et nucléaire (Yamaji 2015 : 59; Kimura: 2009), puis à l'augmentation des achats de charbon et de gaz naturel liquéfié (fig. 1).

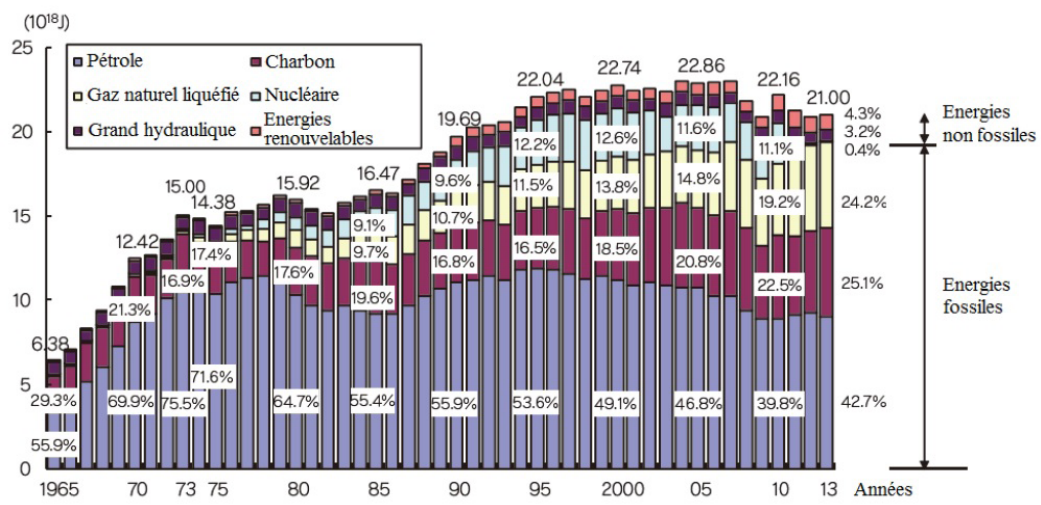

Fig. 1

Composition du mix énergétique par source au Japon de 1965 à 2013.

Source : METI 2015a : 109. Traduit et adapté par Benoit Granier. 
Ce contexte de crise énergétique déclenche en outre le début de la politique shōene, principalement à destination du secteur industriel. Comme l'explique Kimura (2009), le programme Moonlight (Münraito keikaku ムーンライト計画), mis en place en 1978, prévoit d'améliorer l'efficacité énergétique de l'industrie grâce à des avancées technologiques (batteries de stockage, systèmes de cogénération, etc.). L'adoption de la loi $\mathrm{n}^{\circ} 49 \mathrm{du}$ 22 juin 1979 sur l'usage rationnel de l'énergie (Enerugì no shiyō no gōrika nado ni kansuru hōritsu エネルギーの使用の合理化等に関する法律), ou loi Shōene (shōene-hō 省エネ法), constitue aujourd'hui encore, après plusieurs révisions, la pierre angulaire de la politique shōene. Elle préfigure en effet des réglementations contraignantes dans l'industrie.

Dans le même temps, des campagnes de sensibilisation sont organisées auprès des citoyens. Le gouvernement du Premier ministre Tanaka Kakuei 田中角栄 (1972-1974) demande expressément à la population de ne pas gaspiller l'énergie (Garon 1997 : 174). Instauré en avril 1974, le Conseil central de coordination des campagnes populaires de valorisation des ressources et de l'énergie mène des campagnes de communication en faveur de la frugalité et des modes de vie économes en énergie (Garon 1997 : 174-175). Il mobilise les associations de voisinage (chōnaikai 町内会) et de femmes au foyer, les collectivités locales et les écoles, tandis que des ballons publicitaires incitant à limiter les dépenses d'énergie parcourent le ciel des grandes villes. À ces campagnes s'ajoute la consécration symbolique de périodes de l'année au shōene. Février devient en 1976 "mois shōene" tandis que le premier jour de chaque mois est désigné « journée shōene » à partir de 1980. La même année, le $1^{\text {er }}$ août et le $1^{\text {er }}$ décembre deviennent également des "journées de révision générale du shōene": collectivités locales et entreprises sont chargées de mener des activités d'éducation et de sensibilisation (JECH 2014). Après le second choc pétrolier, le Premier ministre Ōhira Masayoshi 大平正芳, avec plusieurs membres de son cabinet et du Parti libéral-démocrate (Jimintō 自民党), adoptent un « look shōene » (shōene rukku 省エネルック) pour inciter les employés de bureau à porter des chemises et vestes à manches courtes et/ou à retirer veste et cravate afin de réduire l'usage de la climatisation (Hagiwara 2006).

Intenses pendant les quelques années suivant chaque choc, les campagnes gouvernementales déclinent néanmoins dès les années 1980, marquées par le gonflement de la bulle spéculative financière et immobilière et, comme ailleurs dans le monde, par le contre-choc pétrolier (Garon 1997 : 
176-177). La question de la demande en énergie des ménages est alors considérée sous l'angle de l'amélioration de la performance des équipements domestiques, plutôt que celui du changement des comportements (JECH 2014 : 23).

Ainsi, la politique shöene ne concerne dans un premier temps que très peu le secteur résidentiel, l'objectif n'étant pas de réduire la demande en énergie des ménages mais de faire face à sa hausse attendue dans un contexte de croissance économique soutenue (Oshitani 2006 : 121). En outre, il s'agit davantage d'accroître l'efficacité énergétique que d'économiser l'énergie en s'adressant aux pratiques quotidiennes des ménages. Ainsi, dans les années 1970, l'objectif de changer les comportements est encore modeste et avant tout conjoncturel. Les premières révisions de la loi Shöene de 1983 et 1993 n'intégrant pas davantage cet enjeu, la situation perdure jusqu'à la fin des années 1990.

\section{Le changement des comportements, un enjeu reconfiguré par la lutte contre le changement climatique}

À partir des années 1990, la consommation d'énergie issue du secteur résidentiel $^{7}$ et le comportement des ménages gagnent en importance pour deux raisons. Le gouvernement n'a plus en effet pour seul but de garantir la sécurité énergétique du pays et la compétitivité des firmes de l'archipel : le volontarisme du Japon dans la lutte contre le changement climatique ${ }^{8}$, la réduction de la demande en énergie et son amélioration deviennent cruciaux pour réduire les émissions de $\mathrm{CO}_{2}$ (METI 2014; Oshitani 2006).

7. Les statistiques énergétiques officielles du gouvernement japonais regroupent les secteurs tertiaire et résidentiel dans une unique catégorie. Par souci de clarté, nous suivons plutôt la distinction opérée entre le secteur résidentiel au sens restreint de l'habitat de l'ensemble des ménages (katei bumon 家庭部門) et le secteur tertiaire constitué par les bureaux et les commerces (gyōmu bumon 業務部門) également employée par le METI. À noter que la consommation d'énergie liée aux transports n'entre pas dans ces catégories. 8. Dont témoignent la participation du Premier ministre de l'époque, Takeshita Noboru 竹下登, à la conférence de Toronto sur le réchauffement climatique de 1988, puis l'organisation de la troisième Conférence des parties à Kyoto en 1997. 
D'autre part, la demande en énergie liée au secteur résidentiel augmente continuellement, au point qu'elle aurait plus que doublé entre 1970 et 1990, selon le Japanese Energy Conservation Handbook de 2014 (JECH 2014: 73; fig. 3). Le METI explique cette hausse par l'instauration de modes de vie énergivores fondés sur la recherche du confort et de la commodité, se traduisant par la multiplication des appareils électroménagers dans les foyers ${ }^{9}$ (JECH 2014 : 2-3). On observe en particulier une très forte hausse du taux d'équipement en climatiseurs (Wilhite et al. 1996).

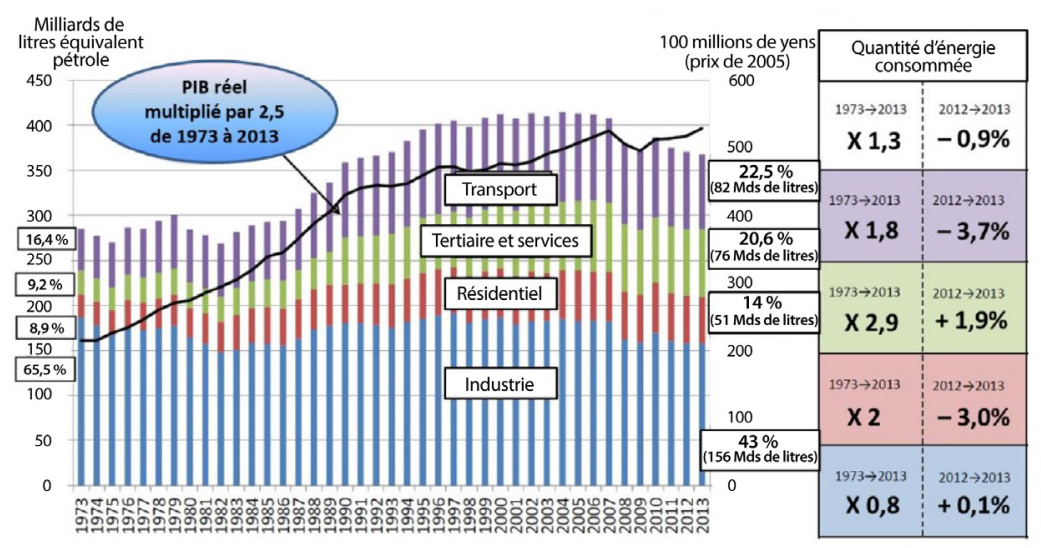

Fig. 2

Consommation d'énergie par secteur au Japon de 1973 à 2013.

L'inscription à l'agenda politique de la question climatique et la hausse de l'énergie consommée par le secteur résidentiel ont alors deux incidences importantes. La première concerne la manifestation des intérêts du MITI et de ses partenaires du secteur privé, représentés en particulier par le

9. Ce constat est dressé dès 1993 par le directeur de l'agence pour les ressources naturelles et l'énergie (Shigen enerugī chō 資源エネルギー庁) du MITI, Kuroda Naoki 黒田直樹, devant la commission de l'industrie, des ressources naturelles et de l'énergie (Sangyō shigen enerugī ni kansuru chōsakai 産業・資源エネルギーに関する調査会) de la Chambre des conseillers ( $8^{\mathrm{e}}$ réunion de la $126^{\mathrm{e}}$ assemblée, le 12 mai 1993). 
Keidanren. Selon le ministère et ces derniers, en effet, l'industrie japonaise présentait alors le meilleur niveau d'efficacité énergétique au monde (Sawa 2010; Oshitani 2006 : 112) : elle ne pouvait donc pas faire davantage d'efforts au risque d'entraver gravement sa compétitivité. Par conséquent, les progrès devaient être obtenus du secteur résidentiel, ce qui impliquait d'agir sur le comportement des ménages - en particulier sur leurs achats d'appareils (Oshitani 2006: 247). La promotion des comportements shōene vise ainsi à contenir la demande en énergie de l'archipel en épargnant l'industrie. Toutefois, le MITI n'introduit pas de nouvelles mesures dans un premier temps, à l'exception d'un système de labellisation (shōene raberingu seido 省エネラベリング制度), qui impose aux détaillants d'afficher des informations sur l'efficacité énergétique de l'appareil. Figurent ainsi les performances relatives et absolues du modèle, accompagnées d'une vignette de couleur, orange ou verte, si l'efficacité énergétique est inférieure ou supérieure au standard fixé. Suite à la révision de la loi Shōene de 2002, une dimension comparative est ajoutée : une évaluation sur un barème de cinq étoiles situe l'appareil vis-à-vis des autres produits et marques de sa catégorie (fig. 3) (JECH 2014 : 71-72).

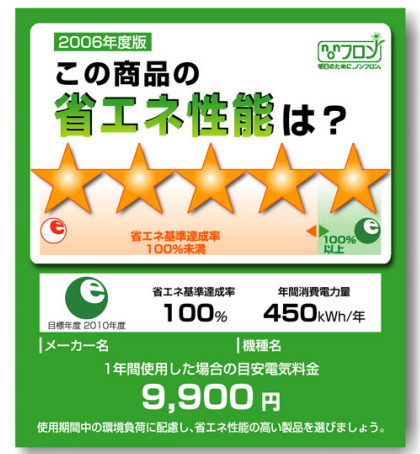

Fig. 3

Label sur l'efficacité énergétique.

Source : JECH 2014 : 71.

La deuxième incidence est l'irruption d'un nouvel acteur dans le gouvernement des conduites consommatrices d'énergie. Comme expliqué plus haut, le MITI était à l'origine le seul ministère compétent en matière de 
politique énergétique et, par conséquent, de changement des comportements. En effet, l'agence de l'Environnement était initialement chargée des problèmes de pollution, de dégradation du paysage et d'accumulation des déchets. À partir des années 1990 néanmoins, elle commence à se préoccuper des enjeux d'environnement global (chikyū kankyō 地球環境), au premier rang desquels le réchauffement climatique ${ }^{10}$ (Oshitani 2006 : 79-81). L'agence devient alors un nouvel acteur de la politique énergétique en raison du couplage des enjeux énergétiques et climatiques ${ }^{11}$. Pour elle, l'ampleur des réductions nécessaires implique des efforts considérables dans tous les secteurs, en particulier résidentiel. Dès 1990, elle propose un plan d'action pour mettre en place des modes de vie peu émetteurs de $\mathrm{CO}_{2}$, caractérisés par une réduction du temps de travail, une moindre utilisation de la climatisation et du chauffage, un changement d'heure saisonnier et la promotion d'appareils efficients (Oshitani 2006 : 102-103; Nishioka \& Morita 1992). Toutefois, à l'exception des deux dernières ${ }^{12}$, ces propositions ne sont pas retenues par le MITI et le gouvernement japonais.

Quelques semaines avant la conférence organisée à Kyoto en novembre 1997, l'agence délivre sa première campagne nationale de sensibilisation et d'engagement contre le changement climatique. Intitulée Ekoraifu hyakuman-nin no chikai エコライフ100万人の誓い (Eco-life : le serment d'un million de personnes pour une vie écologique) ou Eco-Life Pledge, la campagne visait à obtenir l'engagement d'un million de personnes à adopter autant que possible douze comportements respectueux de l'environnement. La moitié d'entre eux concerne la consommation d'énergie : maintenir la température intérieure en dessous de $20^{\circ} \mathrm{C}$ l'hiver et au-dessus de $28^{\circ} \mathrm{C}$ l'été, bien éteindre les lumières et débrancher les appareils, ou

10. Les problèmes environnementaux étaient jusqu'alors limités au territoire national, à l'image de la gestion des pollutions industrielles et des déchets. Les mises à l'agenda international, puis sur celui du gouvernement japonais, des questions d'environnement global a par la suite élargi le champ d'action de l'agence.

11. Puisque la réduction des émissions de $\mathrm{CO}_{2}$ passe en majeure partie par la hausse de l'efficacité énergétique, la diminution de la demande en énergie et la décarbonisation du mix énergétique.

12. La tentative d'instaurer l'heure d'été a cependant échoué à cause de son impopularité : elle était associée à un système similaire, mis en place pendant l'occupation étasunienne (1945-1952), ayant allongé les horaires de travail (Oshitani 2006 : 113). 
économiser l'énergie en faisant la cuisine ${ }^{13}$ (MOE 1997). Créé par l'agence en 1997, le Quartier général pour la prévention du réchauffement climatique (Chikyū ondanka taisaku suishin honbu 地球温暖化対策推進本部) consacre une partie de ses Directives sur les mesures de prévention du réchauffement climatique (Chikyū ondanka taisaku suishin keikaku sakutei gaidorain 地球温暖化対策推進計画策定ガイドライン) de 1998 à la « révision des modes de vie» (raifusutairu no minaoshiライフスタイルの見直し) (Kantei 1998). Les principaux moyens envisagés sont l'information et la sensibilisation de la population aux enjeux énergétiques et climatiques, à travers l'éducation et des campagnes de communication, avec un accent sur la promotion des logements, équipements et véhicules à faible dépense énergétique. Le document préconise à nouveau l'adoption de mesures dites shōene, comme le réglage du thermostat, ou de "nouveaux styles vestimentaires tendance " (aratana ryūkō no yōshiki 新たな流行の様式) et de "nouveaux modes de vie à faible coût énergétique " (shöenerugi-gata no atarashii raifusutairu 省エネルギー型の新しいライフスタイル). Toutefois, ce programme d'action ne mène, dans l'immédiat, à aucune mesure concrète en dehors des campagnes initiées l'année précédente.

\section{Les moyens de communication du $\mathrm{xxI}^{\mathrm{e}}$ siècle au service du changement des comportements : de Team Minus $6 \%$ à Fun to Share}

Après la révision des Directives sur les mesures de prévention du réchauffement climatique et l'instauration d'une nouvelle campagne adoptant le même registre en 2002, le nouveau ministère de l'Environnement défend un programme de sensibilisation d'une envergure inédite en 2005, année de l'entrée en vigueur du Protocole de Kyoto. En étroite collaboration avec la deuxième agence publicitaire du Japon, Hakuhōdō 博報堂, il inaugure au mois d'avril l'initiative "Tous ensemble, stoppons le réchauffement. L'équipe des moins $6 \%$ " (Minna de tomeyō ondanka chìmu mainasu roku

13. Par exemple: privilégier les cuissons au four à micro-ondes, cuisiner en grande quantité puis réchauffer ou manger froid, prendre les repas en famille, etc. 
pāsento みんなで止めよう温暖化 チーム・マイナス6\%) ou Team Minus $6 \%$ (MOE 2005a). Cette dernière vise à susciter un esprit d'équipe pour impliquer tous les acteurs (gouvernement, citoyens, entreprises et société civile) dans la réduction de $6 \%$ des émissions de $\mathrm{CO}_{2}$ assignée par le Protocole. Un site internet ${ }^{14}$ permet à chacun de devenir membre de l'équipe et d'obtenir une reconnaissance de ses efforts. Sur internet, dans la presse et à la télévision, Team Minus $6 \%$ soutient six actions concrètes contre le réchauffement : fixer le climatiseur à $28^{\circ} \mathrm{C}$ (minimum) l'été et le chauffage à $20^{\circ} \mathrm{C}$ (maximum) en hiver; pratiquer un mode de conduite (automobile) shōene; toujours fermer les robinets après usage; choisir des produits respectueux de l'environnement; débrancher les appareils non utilisés; refuser le packaging excessif ${ }^{15}$ (MOE 2005a).

Dans le même temps, Koike Yuriko 小池百合子, alors ministre de l'Environnement, s'investit personnellement dans la mise en place d'une paire de mesures particulièrement innovantes, Cool Biz (küru bizu クールビズ) et Warm Biz (wōmu bizu ウォームビズ). S’adressant aux employés de bureau des secteurs privés et publics, elles consistent à promouvoir certains comportements en agissant sur les normes sociales, c'est-à-dire ce qui est considéré comme socialement acceptable et pertinent. Lors de la première campagne Cool Biz de l'été 2005, les employés du service public sont tenus de limiter l'usage du climatiseur de façon à ce que la température intérieure ne soit pas artificiellement réduite à moins de $28{ }^{\circ} \mathrm{C}$; les employés du secteur privé sont quant à eux sommés d'opter pour une chemise à manches courtes, sans veste ni cravate (MOE 2005b).

Le MOE recommande à son tour aux entreprises privées et à leurs salariés de faire de même, avec le soutien de la Fédération des organisations économiques japonaises (Nippon keizai dantai rengō-kai 日本経済団体連合会 ou Nippon keidanren) et de nombreuses firmes emblématiques telles les groupes Toyota (Toyota jidōsha kabushiki gaisha トヨ夕自動車株式会社) et la chaine de grands magasins Mitsukoshi (Kabushiki gaisha Mitsukoshi 株式会社三越). En outre, plusieurs membres du gouvernement et chefs d'entreprises éminents adoptent eux-mêmes, dans les médias et à l'occasion

14. Site accessible à l'adresse : http://www.team-6.jp/.

15. Ces six actions figuraient déjà dans les douze conduites préconisées par l'Eco-Life Pledge de 1997. 
de défilés de mode, ce code vestimentaire afin que le style décontracté au travail soit socialement mieux toléré et même valorisé (MOE 2005c). Le Premier ministre Koizumi Jun.ichirō 小泉純一郎 revêt publiquement une tenue Cool Biz très médiatisée, pour montrer l'exemple et remettre en question l'étiquette japonaise imposant veste et cravate sur la plupart des lieux de travail (Slade 2009). Dans la même optique, Warm Biz incite à porter des vêtements chauds en hiver afin de limiter la consommation d'énergie liée au chauffage.

Si Warm Biz a été peu étudié et semble peu efficace, Cool Biz est communément considéré comme un grand succès - par ailleurs inhabituel dans ce domaine - et aurait largement contribué à l'évolution du code vestimentaire des employés de bureau (Tan et al. 2008 : 12; Sunikka-Blank \& Iwafune 2011).

À partir d'octobre 2006, ce type d'action est étendu au secteur domestique dans le cadre du programme Uchi-eco (内エコ), qui promeut des pratiques écologiques en matière d'habillement, d'alimentation, de chauffage... À titre d'exemple, lors de l'hiver 2007, le slogan «Si l'on se rassemble tous, Uchi-eco!» (Minna de atsumareba Uchi-eco! みんなで集まれば うちエコ!) invitait les amis et les familles à se réunir plus souvent afin de réduire l'espace à chauffer en hiver ou à rafraîchir en été (MOE 2006a). La campagne "Je déclare la réduction d'un kilogramme de $\mathrm{CO}_{2}$ par jour et par personne ${ }^{16} "$, qui accompagne Team Minus $6 \%$ et Uchi-eco, renseigne en outre sur les effets concrets des comportements à adopter grâce à un système de quantification : régler l'air conditionné à $28^{\circ} \mathrm{C}$ ou davantage permet de réduire les émissions de $\mathrm{CO}_{2}$ d'au moins $16,8 \mathrm{~kg}$ chaque été, laver tous les vêtements en même temps économise 3,3 kg par an, remplacer les ampoules incandescentes ou halogènes par des diodes électroluminescentes 46,6 kg, etc. (Tan et al. $2008: 14$ ).

Si les résultats de la campagne Team minus $6 \%$ sur la réduction des émissions de $\mathrm{CO}_{2}$ demeurent difficiles à chiffrer, elle est considérée comme un grand succès en raison de sa notoriété auprès de la population (Sanpei \& Aoyagi-Usui 2009; Tan et al. 2008) et aurait fortement contribué à l'assouplissement du code vestimentaire des employés de bureau (Tan et al. 2008 :

16. D'après le calcul selon lequel une telle réduction permettrait d'atteindre l'objectif des $6 \%$. 
12; Sunikka-Blank \& Iwafune 2011). Entre mai 2005 et juin 2008, plus de 2300000 individus et 21795 organisations publiques et privées ont participé à Team Minus $6 \%$. Parmi les $80 \%$ environ des Japonais qui s'y montraient favorables, $50 \%$ ont affirmé le mettre en œuvre (Tan et al., 2008 : 16-17). Face au constat d'échec de la plupart des campagnes de mobilisation sur le changement climatique, la réussite de Team Minus $6 \%$ aurait donc résidé dans sa capacité à rendre l'enjeu plus concret, d'une part en le reliant à des pratiques aisées à réaliser au quotidien, d'autre part en faisant écho à des valeurs et des traditions familières, comme la pratique de l'uchimizu 打与水, la dégustation d'un nabe 鍋 en famille ${ }^{17}$ ou la référence au concept de mottainai もつたいない ${ }^{18}$. 96,1\% des Japonais connaissaient le programme Cool Biz en 2006 et 43,2\% des entreprises et administrations déclaraient avoir respecté la consigne des $28{ }^{\circ} \mathrm{C}$ au cours de l'été, suscitant d'après le MOE une économie de 1,14 million de tonnes de $\mathrm{CO}_{2}$ (MOE 2006b).

Devenue Super Cool Biz (sūpā kūru bizu スーパークールビズ) en 2011, la campagne a demandé des efforts accrus aux employés suite à l'accident de Fukushima ${ }^{19}$. Le programme Team Minus $6 \%$ a quant à lui été révisé au fil des années et a plusieurs fois changé de nom, s'intitulant Challenge 25 Campaign (charenji nijügo kyanpēn チャレンジ25キャンペーン) à partir de 2010 - pour refléter l'engagement du Premier ministre Hatoyama Yukio 鳩山由紀夫 de réduire les émissions de $25 \%$ d'ici à 2020 - et Fun to Share ${ }^{20}$ depuis 2014. Encore active aujourd'hui, la campagne insiste sur le partage et appelle, plus fortement qu'Uchi-eco, à se rassembler dans des endroits climatisés en été et chauffés en hiver, à travers les volets Cool Share ( $k \bar{u} r u$ shea クールシェア) et Warm Share (wōmu shea ウォームシェア). Ces derniers s'adossent à un site internet et à une application smartphone qui recensent

17. L'uchimizu consiste à récupérer et répandre les eaux usées dans son environnement proche afin de provoquer un rafraîchissement; le nabe désigne une sorte de fondue composée de légumes et de viande et/ou de poisson.

18. L'expression mottainai renvoie à l'idée de gâchis et décrit en particulier le gaspillage alimentaire ou de ressources naturelles et énergétiques.

19. La liste des vêtements acceptables pour aller travailler (certains tous les jours, d'autres uniquement en cas de forte chaleur) a été étendue au port du short, du polo, des baskets voire des sandales, sans rencontrer beaucoup de succès néanmoins.

20. En anglais et italiques dans le texte. 
sur une carte les endroits frais ou chauds ouverts au public, à proximité de l'usager ${ }^{21}$.

Ces programmes révèlent l'intérêt grandissant de l'action publique japonaise pour le secteur résidentiel et les conduites des ménages à partir de 2005, suite à l'émergence d'un nouvel acteur : le MOE. Cette inflexion s'explique par la prégnance des enjeux climatiques et par le constat de la hausse de la demande en énergie du secteur résidentiel, déjà formulé au cours des années 1990. Mais l'intensité des campagnes de communication du MOE s'explique également par son incapacité - en raison de la prédominance du METI - à imposer des réglementations contraignantes aux acteurs économiques privés et à modifier le mix énergétique. Team Minus $6 \%$ et ses successeurs ambitionnent de changer les comportements à travers la sensibilisation et l'exhortation, en utilisant les médias traditionnels (journaux, télévision et radio) mais aussi de nouveaux outils comme Internet et les smartphones. Les comportements visés par ces programmes sont très variés, puisqu'ils regroupent des pratiques aussi bien quotidiennes (réglage du thermostat, style vestimentaire, etc.) que ponctuelles (achat d'appareils économes). L'adoption des programmes d'écopoints et d'Uchi-eco shindan par le MOE en 2009 et 2011 met en place la diffusion de ces pratiques (encadré $n^{\circ} 1$ ).

21. Site accessible à l’adresse : http://sharemap.jp/. 


\section{Encadré $n^{\circ} 1$ : Les programmes d'écopoints (2009-2014) et Uchi-eco shindan}

En mai 2009, le MOE a inauguré le dispositif des écopoints (Eko pointo no katsuyō ni yoru gurīn kaden fukyū sokushin jigyō エコポイントの活用によるグリーン家電普及促進事業) pour stimuler l'économie et favoriser la dissémination des appareils électroménagers efficients (METI 2011). L'achat de modèles de téléviseurs, de climatiseurs et de réfrigérateurs avec une performance labellisée quatre ou cinq étoiles entre mai 2009 et mars 2011 rapportait au consommateur plusieurs milliers d'écopoints, échangeables contre des produits labellisés shōene, des coupons ou des cartes prépayées. $82 \%$ des points délivrés l'ont été pour l'achat d'un téléviseur, en raison du grand nombre de points à la clef (METI 2011). II a en outre été élargi à la construction de logements efficients et à la rénovation du bâti par le MOE, conjointement avec le METI et le MLIT. Mis en place pour répondre à la crise des subprimes, ce programme d'écopoints dédié au logement a été réitéré pour soutenir la reconstruction du Tōhoku à partir de 2011 et la revitalisation régionale à partir de 2014 (Tōyō Keizai 2015).

Dans le même temps, le MOE a mis en œuvre, entre les années fiscales 2011 et 2014, le programme de diagnostic énergétique Katei eko shindan seido 家庭工コ診断制度, dit Uchi-eco shindan. Des spécialistes certifiés par le ministère étaient chargés de réaliser des diagnostics au domicile des ménages volontaires, qui bénéficiaient de recommandations et de subventions pour améliorer l'efficacité énergétique de leur logement. Plusieurs études suggèrent que les foyers ayant bénéficié de ces diagnostics, motivés par la réduction de leur facture en gaz et/ou en électricité, ont significativement réduit leur consommation d'énergie.

En résumé, les dispositifs de gouvernement des conduites déployés par le METI et le MOE avant la décennie 2010 répondaient globalement à deux logiques. La première est d'ordre économique : elle mise sur la capacité des consommateurs à traiter l'information et à choisir les produits de manière à maximiser leurs gains (labellisation, écopoints, Uchi-eco shindan). La seconde repose sur la sensibilisation et la responsabilisation : elle suppose que l'engagement des citoyens à lutter contre le changement climatique les conduit à adopter des comportements shöene. Ces logiques ont deux points communs. D'une part, elles sont fondées sur l'hypothèse que les individus agissent de manière rationnelle en fonction, soit de leur volonté de maximiser leurs gains, soit de leurs valeurs et engagements. Cette logique renvoie au modèle de changement des comportements qui domine dans la plupart des pays de l'OCDE, en particulier dans le domaine de l'énergie et du changement climatique (Shove 2010). D’autre part, elles se matérialisent par des instruments génériques qui transmettent une information identique à l'ensemble de la population, à l'exception d'Uchi-eco shindan 
qui fournit, à petite échelle, des conseils personnalisés aux ménages. Ces logiques et leurs fondements sont toutefois partiellement remis en question dans les années 2010 : de nouveaux types d'instruments voient le jour dans un contexte marqué par l'accident de Fukushima et la libéralisation des marchés de l'énergie.

\section{De nouveaux instruments fondés sur les sciences comportementales et le big data}

La politique shōene connaît en effet à partir des années 2010 un infléchissement remarquable avec le recours à des instruments fondés sur les sciences comportementales. Ces dispositifs inédits ont d'abord été conçus et appliqués aux États-Unis, en se nourrissant des avancées en psychologie et en économie comportementale et de l'essor du big data et des nouvelles techniques de mesure de la consommation d'énergie. Le METI et le MOE se sont à leur tour approprié ces instruments dans un contexte marqué par la catastrophe de mars 2011, la mise en concurrence des fournisseurs d'énergie et l'urgence représentée par le réchauffement climatique.

\section{L'essor international des sciences comportementales et leur mobilisation dans les politiques de gouvernement des conduites au Japon}

Au cours des années 2000, les deux logiques exposées plus haut, prédominantes dans l'ensemble des pays de l'OCDE, sont de plus en plus critiquées. D’une part, les travaux de recherche en sciences humaines et sociales fournissent de plus en plus d'éléments montrant que les individus ne se comportent souvent ni en accord avec leurs valeurs et leurs engagements, ni de manière à maximiser leur intérêt économique. Plusieurs chercheurs en économie comportementale et en psychologie, qui s'attachent à identifier les biais rendant le comportement humain "irrationnel ", reçoivent alors 
des prix prestigieux pour leurs travaux ${ }^{22}$. D'autre part, les politiques de changement des comportements s'avèrent d'une efficacité limitée et leur conception erronée des réactions humaines est fortement mise en cause (Shove 2010).

Dans ces conditions, plusieurs États décident de solliciter des spécialistes d'économie comportementale et de psychologie pour élaborer des politiques plus efficaces. Ce phénomène survient dans la continuité de la publication du best-seller Nudge: Improving Decisions About Health, Wealth, and Happiness, écrit par les universitaires états-uniens Richard Thaler et Cass Sunstein (Thaler \& Sunstein 2008). Nudge signifie " coup de pouce " et le verbe to nudge renvoie au fait de pousser doucement du coude ou d'encourager. Les deux auteurs le définissent comme une action sur l'environnement des individus en vue d'influencer leur comportement de manière prévisible, sans coercition ni recours explicite à des signaux économiques. Sur la base des résultats obtenus par les sciences comportementales, ils proposent une "troisième voie ", entre interventionnisme étatique et laisserfaire, qui oriente les comportements sans les contraindre, en prenant en compte leur dimension irrationnelle mais néanmoins prédictible.

La doctrine des nudges a dépassé la sphère des débats académiques lorsque Sunstein a été nommé en 2009 à la tête de l'Office of Information and Regulatory Affairs aux États-Unis, une année avant que Thaler ne rejoigne la Behavioural Insights Team du gouvernement britannique, également connue sous le nom de Nudge Unit. Elle s'est ainsi matérialisée à travers de nombreux dispositifs d'action publique aux États-Unis et au Royaume-Uni, mais aussi dans d'autres pays de l'OCDE (Allemagne, Australie, Danemark, entre autres). Les sciences comportementales et les nudges jouissent d'une grande popularité auprès des acteurs des politiques énergétiques en particulier, surtout aux États-Unis. De nombreux États instaurent ainsi des dispositifs de changement des comportements calés sur le modèle des Home Energy Reports (HER) publiés par la firme états-unienne

22. La médaille John Bates Clark est décernée à Matthew Rabin en 2001 et à Steven Levitt en 2003, tandis que le prix Nobel d'économie est attribué à Daniel Kahneman en 2002 pour avoir introduit en sciences économiques des acquis de la recherche en psychologie. Les économistes situés dans ce champ ont fondé une branche de la discipline, qui a pris le nom d'économie comportementale. 
Opower ${ }^{23}$. Ces derniers s'appuient sur les résultats des sciences comportementales et sur les progrès accomplis dans la récolte et l'analyse des données de consommation d'énergie des ménages, pour optimiser le contenu et la forme de l'information qui leur est transmise ${ }^{24}$. Les HER contiennent ainsi des indications comme :

- la comparaison entre la consommation mensuelle du ménage, la consommation moyenne d'un échantillon de foyers de taille similaire, et la consommation des foyers les plus économes (jeu sur la tendance à se conformer à la norme sociale);

- la représentation graphique de la consommation annuelle du ménage, mois par mois, en comparaison avec la moyenne des autres foyers et avec celle des plus économes, accompagnée d'un message du type : "cette année, vous avez perdu 150 euros par rapport à des foyers similaires qui consomment moins" (car mettre en avant les pertes effectives a plus d'impact que l'évocation des gains potentiels);

- la comparaison entre la consommation mensuelle du ménage et sa consommation durant le même mois de l'année précédente, avec un émoticône pour l'encourager à s'améliorer ;

- des conseils personnalisés et illustrés pour réduire sa consommation d'énergie, dont la combinaison s'appuie sur des théories psychologiques.

\section{L’application des nudges par la politique shōene japonaise}

C'est dans ce contexte international que survient la triple catastrophe de mars 2011. L'arrêt des réacteurs nucléaires rend encore plus urgent le changement des comportements. En outre, la catastrophe révèle la vulnérabilité du réseau électrique et remet en question le fonctionnement du marché et de ses principaux acteurs : leur gestion de la crise est jugée désastreuse par

23. Créée en 2007 et rachetée par Oracle en 2016, Opower était une start-up spécialisée dans le développement de services d'engagement client à destination des fournisseurs d'énergie.

24. La diffusion des compteurs communicants et des techniques de traitement des données massives (big data) ont en effet permis à Opower de bâtir une plateforme technologique capable d'analyser chaque année plus de 300 milliards de données issues de relevés de compteurs (Cuddy et al. 2010). 
l'opinion et les observateurs. Cela conduit au vote d'un projet de réforme du marché de l'électricité en novembre 2013, dont la révision de juin 2014 promulgue la mise en concurrence des fournisseurs d'électricité à partir de $2016^{25}$. Ces fournisseurs se préoccupent désormais d'offrir des prestations de service pour économiser l'énergie, sous la pression du METI mais aussi dans l'optique de conserver leur clientèle (Nishimura 2014).

Dès l'été 2013, deux compagnies d'électricité expérimentent de tels services. Tōkyō denryoku 東京電力 $\left(\mathrm{TEPCO}^{26}\right)$ signe au mois d'octobre un partenariat commercial avec Opower, qui vient d'ouvrir un bureau à Tokyo. Les clients de la région du Kantō peuvent ainsi accéder à des conseils personnalisés à partir du portail web de leur fournisseur d'électricité. Quant à Kansai denryoku 関西電力 $\left(\mathrm{KEPCO}^{27}\right)$, elle propose des consultations shōene (shōene konsaru 省エネコンサル) et des conseils personnalisés (shōene adobaisu 省エネアドバイス) à 341 ménages participant à une expérimentation que finance le $\mathrm{METI}^{28}$. Les entretiens (d'une heure à une heure trente) se structurent autour d'un document remis aux ménages, largement inspiré des rapports d'Opower (Nishimura 2014). Ils ont toutefois pour originalité d'assimiler la courbe-type de consommation des ménages à un animal, comme l'illustre la figure 4.

25. Au moment de l'accident de Fukushima, en dépit des réformes de libéralisation votées depuis les années 1990, le marché de l'électricité reste largement contrôlé par dix monopoles régionaux privés, qui cumulent des activités de production, de distribution/ transport et de commercialisation de l'électricité.

26. Kansai Electric Power Company.

27. Tokyo Electric Power Company.

28. KEPCO prend cette initiative au sein de la smart community (sumāto komyuniti スマートコミュニティ) de Kyoto Keihanna (Keihanna eko shiti けいはんなエコシティ), sélectionnée par le METI dans le cadre du programme " Démonstrateurs d'énergies de nouvelle génération et de systèmes sociaux " (Jisedai enerugī shakai shisutemu jisshō jikken 次世代エネルギー・社会システム実証実験). Pour davantage de détails sur les dispositifs introduits dans les smart communities, voir Granier (2015). 

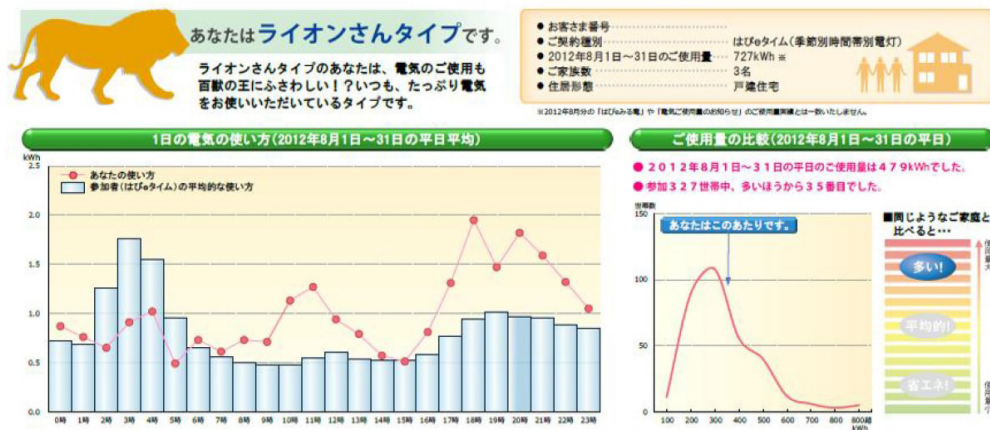

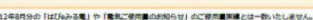

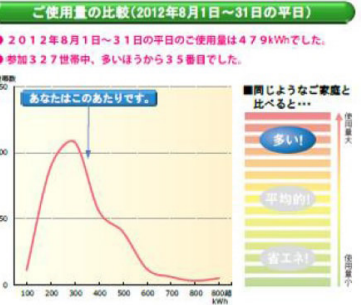

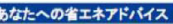

無理な努力をしなくても、ほんの少し家電機器の使いちを見直すだけでググッと 省エネ! ? 光敏費を大きく削減できるかも!

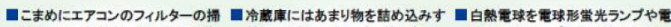 除をしていただと、ほこりの目詰 ぎないようにしましょう。整いもの 球形LEDランブに取り替えていた \\ 末りによる効萧低下を防ぐことがて

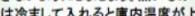 \\ だくと、省エネになります。}

き、畄エネになります。

がらず省エネにななりま。

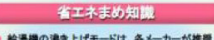

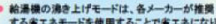
t5.

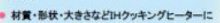

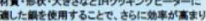

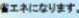

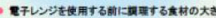

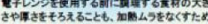
leuriser.

Fig. 4

Feuillet «Vous êtes de type Lion » (Anata wa raionsan taipu desu あなたはライオンさん タイプです).

Document remis en main propre par le bureau de KEPCO Fushimi.

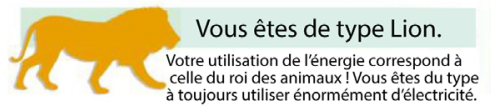

- Tyme de contrat Hapi « e s time

- Électrictéconsommée en aout 2012 Hapi a 727 hi

Nombre de personnes dans le foyer 3

Typed'habitation à toujours utiliser énormément d'électricité.

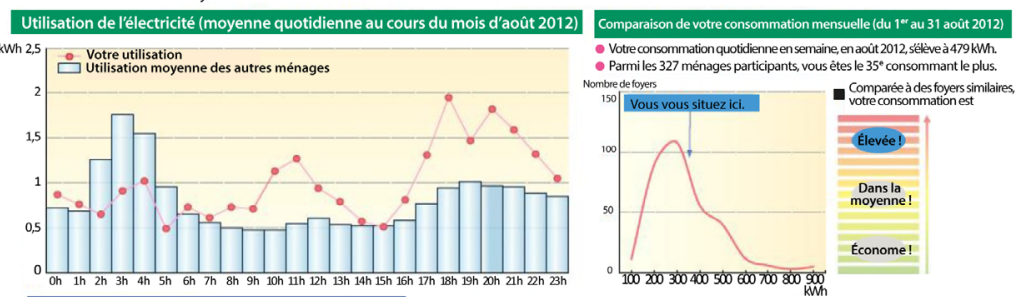

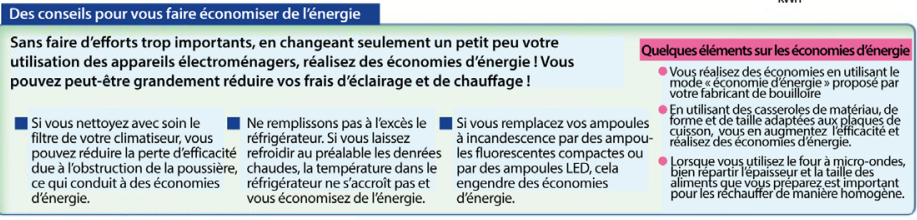

Fig. 4, traduction par Benoit Granier. 
L'année suivante, le Jūkankyō keikaku kenkyūjo 住環境計画研究所 (JYURI), un think tank partenaire de longue date du MOE et du METI - son PDG, Nakagami Hidetoshi 中上英俊, est président du Comité shōene-, fonde le Shōenerugī kōdō kenkyū-kai 省エネルギー行動研究会 (Society of Energy Efficiency \& Behavior), qui encourage les recherches sur les comportements shōene dans les domaines de l'éducation, de la psychologie et de l'économie comportementale (SEEB 2014). Le groupe d'étude obtient le soutien des acteurs gaziers (Tokyo Gas et Osaka Gas en particulier), du METI et du MOE pour l'organisation de la première conférence BECC Japan (Kiköhendō shōenerugī ködō kaigi 気候変動・省エネルギー行動 会議) en septembre 2014, c'est-à-dire la version locale de la Behavior, Energy and Climate Change Conference qui a lieu aux États-Unis chaque année depuis 2007 (Hirayama 2015). Suite au lobbying du JYURI, le METI ${ }^{29}$ le charge en juillet 2015 d'expérimenter les HER avec Opower Japan et Hokuriku denryoku 北陸電力 $\left(\mathrm{HEPCO}^{30}\right)$ (Denki Shinbun 2015).

Aux fins de mesurer les effets de l'intervention, 40000 foyers ont été recrutés puis répartis aléatoirement selon deux groupes de taille équivalente : un groupe "traitement " à qui les HER sont adressés et un groupe "témoin ". En décembre 2015 et janvier 2016, les 20000 ménages du groupe « traitement » ont reçu des HER sous la forme d'un feuillet A4 rectoverso, sur le modèle états-unien (fig. 5). Cet instrument montre jusqu’à quel point les acteurs de la politique énergétique ont revu leur conception du comportement humain : les individus ne se comportant pas toujours de manière rationnelle, l'intervention sur leurs conduites doit agir sur des ressorts autres que la recherche du gain ou l'engagement à lutter contre le changement climatique. Il reflète également l'impact du développement du big data sur les acteurs publics et leurs partenaires, qui bénéficient désormais des moyens de mesurer les effets et d'optimiser la conception des dispositifs de promotion des comportements shöene.

29. D'autres chercheurs et représentants de think tanks ont préconisé au METI de recourir aux sciences comportementales (Granier 2017, chap. 7).

30. Hokuriku Electric Power Company. 


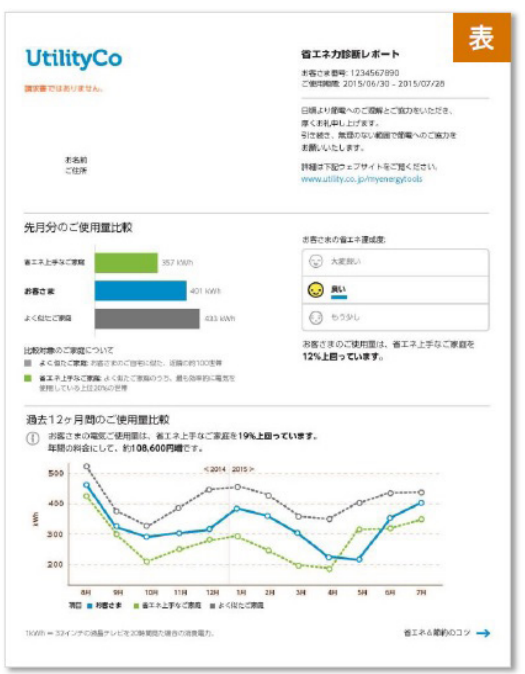

Fig. 5

Les HER du METI.

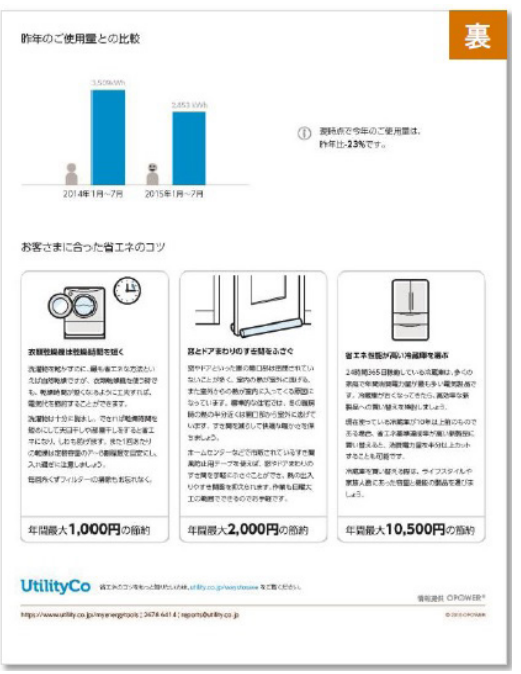

Source : METI 2016.

Quelques mois après l'expérimentation des HER par le METI, le MOE consacre une partie de son budget pour l'année fiscale 2017 à mobiliser les sciences comportementales afin d'influencer les conduites. Deux à trois milliards de yens sont ainsi investis annuellement dans des « initiatives de promotion de mesures spontanées de la part des ménages par l'intermédiaire de la diffusion d'informations encourageant un changement des comportements (nudges) de type bas-carbone " (Teitanso-gata no kōdō hen.yō o unagasu jōhō hasshin (najji) ni yoru katei nado no jihatsuteki taisaku suishin jigyō 低炭素型の行動変容を促す情報発信(ナッジ)による家庭等の自発的対策 推進事業) (MOE 2016 : 32). Selon l'appel à projets :

Il est indispensable de créer des innovations destinées à un changement drastique des comportements vers un type bas-carbone [...]. Ces dernières années, en Europe et aux États-Unis, les politiques gouvernementales ont soutenu des programmes de changement des modes de vie et des comportements individuels, selon une approche fondée sur les sciences comportementales. Leur rentabilité est élevée, et on souligne que cette approche préserve la liberté des destinataires. Cependant, la vérification de leur applicabilité durable dans notre pays est nécessaire. (MOE 2016 : 32) 
L'appel à projets a menéà la sélection de trois programmes d'expérimentation financés sur une durée de cinq ans (2017-2021). Parallèlement, le MOE instaure en avril 2017 la Nihonban najji yunitto (日本版ナッジ・ユニット) ou Nudge Unit of Japan, dans le but de créer un « modèle japonais de changement des comportements " (Nihon-gata no kōdō hen.yō moderu 日本型の 行動変容モデル) s'appuyant sur les nudges et les sciences comportementales (MOE 2017). Cette unité est composée de personnalités issues de l'industrie, du monde académique et des gouvernements centraux et locaux. En effet, l'engouement des acteurs publics pour les nudges ne se limite plus à l'échelon national puisqu'en 2017, le département de l'environnement et de l'énergie du gouvernement métropolitain de Tokyo (Tōkyō-to kankyōkyoku chikyū kankyō enerugī-bu 東京都環境局地球環境エネルギー部) a lui aussi mis sur pied une équipe de spécialistes en sciences comportementales afin de réduire la consommation des ménages sous sa juridiction.

\section{Conclusion}

L'objectif de maîtriser la demande en énergie a conduit le gouvernement japonais à déployer en une quarantaine d'années un nombre croissant de dispositifs ciblant les comportements individuels. Conjoncturel au lendemain des chocs pétroliers, l'intérêt pour la consommation d'énergie des ménages a été renforcé par l'agenda climatique, qui a provoqué l'entrée en scène du MOE, et par l'accident de Fukushima. Face à l'efficacité limitée des premières mesures mises en œuvre, le METI et le MOE ont mobilisé des instruments rendus accessibles par l'évolution des technologies de l'information et de la communication, et des connaissances sur les comportements. Lors de la décennie 2000, des campagnes de communication d'envergure ont ainsi été menées, en complément - et non en remplacement - de l'approche antérieure fondée sur la réponse des consommateurs aux signaux du marché. Dans les années 2010, ces deux ministères recourent également aux apports combinés des sciences comportementales et du big data, déjà utilisés aux États-Unis et en Europe depuis plusieurs années.

Cette accumulation de dispositifs témoigne du rôle central récemment attribué aux ménages japonais dans la réduction de la consommation d'énergie et des émissions de gaz à effet de serre. D'après les parties 
prenantes de la politique shōene, cela s'explique par le fait que les mesures mises en œuvre par l'État japonais depuis les années 1970 faisaient reposer le fardeau sur les acteurs industriels uniquement. Par conséquent, le potentiel d'économies d'énergie semblait élevé dans le secteur résidentiel, et au contraire limité dans l'industrie, où des efforts supplémentaires risquaient d'entraver la compétitivité des entreprises. Il serait à cet égard intéressant de déterminer si le renforcement des mesures ciblant les ménages s'accompagne ou non d'une diminution de la pression sur les acteurs industriels.

Ainsi, si le but du MOE est de mobiliser un levier supplémentaire pour réduire les émissions de gaz à effet de serre, la mise en ouvre de ces nouveaux dispositifs résulte avant tout des intérêts du METI et des acteurs industriels. En effet, il s'agit non seulement de ne pas entraver la compétitivité des entreprises japonaises, mais aussi de favoriser la croissance économique en promouvant l'achat d'appareils efficients par les ménages. Cet objectif s'inscrit dans la continuité de la politique énergético-climatique d'après-guerre pensée par le METI comme un moyen pour développer l'innovation technologique et stimuler l'activité économique (Oshitani 2006; Watanabe 2011), ce qui n'est pas sans poser question sur la capacité de cette stratégie à réduire les émissions de $\mathrm{CO}_{2}$ à la hauteur des objectifs annoncés (Granier 2019).

Enfin, les phénomènes étudiés dans cet article invitent à examiner comment les intérêts industriels et les rapports de force entre ministères, en interaction avec l'évolution des savoirs et des techniques, affectent le gouvernement des conduites dans d'autres domaines de l'action publique japonaise, comme celui de la santé publique, où les enjeux comportementaux et les intérêts industriels sont également très prégnants et connectés (Walravens 2017). 


\section{Bibliographie}

Cuddy Amy, Doherty Kyle \& Bos Maarten 2010

«OPOWER: Increasing Energy Efficiency

through Normative Influence $(A)$ ", Harvard Business School, Case 911-016.

Denki Shinbun 電気新聞 2015 "Hokuriku denryoku eria de shōene chōsa jisshi e » 北陸電力エリアで省エネ 調査実施へ (Vers la réalisation d'une enquête sur les économies d'énergie dans la région alimentée par la Hokuriku Electric Power Company), 16 juillet. http://www.shimbun.denki.or.jp/news/ main/20150717_02.html (dernière consultation le 20 septembre 2019).

Dubuisson-Queluier Sophie (dir.) 2016 Gouverner les conduites, Paris, Presses de Sciences Po.

\section{FUKASAKU Yukiko 2001}

«Energy and Environment Policy Integration: The Case of Energy Conservation Policies and Technologies in Japan », Energy Policy, 23 (12) : 1063-1076.

\section{Garon Sheldon 1997}

Molding Japanese Minds. The State in Everyday Life, Princeton, Princeton University Press.

\section{Granier Benoit 2015}

«L'expérimentation sociotechnique fondée sur les sciences comportementales : un instrument au service de la production de l'acceptabilité sociale? », VertigO la revue électronique en sciences de l'environnement, 15 (3).

http://vertigo.revues.org/16695 (dernière consultation le 20 septembre 2019).

\section{Granier Benoit 2017}

«Circulations transnationales et transformations de l'action publique. La mobilisation des sciences comportementales dans la politique énergétique japonaise ", thèse de doctorat en science politique, université de Lyon.

\section{Granier Benoit 2019}

«Changer les comportements sans changer les modes de vie. Les impasses du tropisme technologique de la transition énergétique au Japon », Lien Social et Politiques, 82 : 27-51.

Hagiwara Go Wayne 萩原豪 2006 «Enerugī kyōiku to jizoku kanō na shakai » エネルギー教育と持続可能な 社会 (Éducation à l'énergie et société durable), Dai jūgokai Nihon enerugì gakkai taikai 第15回日本エネルギー学会 大会 (Actes de la $15^{\mathrm{e}}$ assemblée de la société de l'energie japonaise), 3-4 août.

HARADA Yumiko 原田由美子 2011

"“Shōene rukku" to "kūru bizu” »「省エネ ルック」と「クールビズ」 (《Look shōene » et « Cool Biz »), Alternative Blog, 21 juillet.

http://blogs.itmedia.co.jp/ harada6stars/2011/07/post_84.html (dernière consultation le 20 septembre 2019).

HiRAYAMA Shō 平山翔 2015

« Kōdō hen.yō to shōenerugī » 
行動変容と省エネルギー (Changement de comportement et efficacité énergétique), Enerugi shigen エネルギー 資源, 36 (3) : 162-166.

\section{JECH 2014}

Japan Energy Conservation Handbook 2014, The Energy Conservation Center, Japan.

\section{Kantei 官邸 1998}

Chikyū ondanka taisaku suishin taikō 地球温暖化対策推進大綱 (Principes fondamentaux de la promotion des mesures contre le réchauffement climatique), 19 juin.

http://www.kantei.go.jp/jp/singi/ ondanka/9806/taikou.html (dernière consultation le 20 septembre 2019).

\section{Kimura Osamu 2009}

«The National Programs for Development of Energy Technologies ", SERC Discussion Paper: SERC09007, Central Research Institute of Electric Power Industry.

http://criepi.denken.or.jp/en/serc/research_ re/download/09007dp.pdf (dernière consultation le 20 septembre 2019).

\section{Leprêtre Nicolas 2016}

«Les transformations de l'action publique au prisme des réseaux électriques intelligents. Le cas des expérimentations de smart communities au Japon », thèse de doctorat en science politique, université de Lyon.

\section{LÉvy Christine \& Ribault Thierry (dir.)} 2012

Ebisu. Études japonaises, 47
(Catastrophes du 11 mars 2011, désastre de Fukushima : fractures et émergences).

\section{METI 2011}

Effects of the Home Appliance Eco-Point System Policy. Ministry of Economy, Trade and Industry, 14 juin.

http://s3.amazonaws.com/zanran_storage/ www.meti.go.jp/ContentPages/2513349263. pdf (dernière consultation le 20 septembre 2019).

\section{METI 2014}

Enerugi kihon keikaku エネルギー基本

計画 (Quatrième plan-cadre sur l'énergie). Ministry of Economy, Trade and Industry. http://www.enecho. meti.go.jp/category/others/basic_plan/ pdf/140411.pdf (dernière consultation le 20 septembre 2019).

\section{METI 2015a}

Enerugī hakusho 2015 エネルギー白書 2015 (Livre blanc sur l'énergie), Ministry of Economy, Trade and Industry. http://www.enecho.meti.go.jp/about/ whitepaper/2015pdf/ (dernière consultation le 20 septembre 2019).

\section{METI 2015b}

Chōki enerugījukyū mitōshi 長期 エネルギー需給見通し (Perspective sur l'offre et la demande d'énergie à long terme). Ministry of Economy, Trade and Industry, 27 juillet 2015.

http://www.meti.go.jp/press/ ess $/ 2015 / 07 / 20150716004 / 2015071600$ 4_2.pdf (dernière consultation le 20 septembre 2019). 


\section{METI 2016}

Enerugī shiyō jōkyōtō no jōhō teikyō ni yoru katei no shōenerugī kōdō hen.yō sokushin kōka ni kansuru chōsa エネルギー使用 状況等の情報提供による家庭の

省エネルギー行動変容促進効果に関 する調査 (Étude sur les effets de la prestation d'information relative à l'usage de l'énergie sur la promotion des comportements économes en énergie des ménages), 28 mars.

http://www.meti.go.jp/meti_lib/ report/2016fy/000233.pdf (dernière consultation le 20 septembre 2019).

\section{MOE 1997}

«Eko raifu hyakuman nin no chikai » no jōkyō ni tsuite「エコライフ100万人の誓い」 の状況について (À propos de la situation de «Eco-life Pledge »), 25 novembre. http://www.env.go.jp/press/1203.html (dernière consultation le 20 septembre 2019).

\section{MOE 2005a}

«Chìmu mainasu roku pāsento » no kikkuofu ni tsuite「チーム・マイナス6\%」の キックオフについて (À propos du lancement de «Team Minus $6 \%$ »), 28 avril.

http://www.env.go.jp/press/press. php?serial=5946 (dernière consultation le 20 septembre 2019).

\section{MOE 2005b}

"Natsu no atarashī bijinesu sutairu » aishō happyō oyobi aichikyū-haku kaijōnai de no rokugatsu itsuka kankyōshō kanren ibento ni tsuite「夏の 新しいビジネススタイル」愛称発表及び愛. 地球博会場内での6月5日環境省関連
イベントについて (À propos de l'annonce de l'appellation «nouveau business style de l'été » et de l'événement organisé le 5 juin à l'exposition d'Aichi en lien avec le ministère de I'Environnement), 27 avril.

http://www.env.go.jp/press/press.

php?serial=5936 (dernière consultation le 20 septembre 2019).

\section{MOE 2005c}

Ai Chikyūhaku kaijō de "kūru bizu korekushon"o kaisai 愛·地球博会場で「 クールビズコレクション」を開催 (La tenue de « Cool Biz collection » à l'exposition d'Aichi), 5 juin.

https://ondankataisaku.env.go.jp/funtoshare/ archive/news/tm6/2005/06/collection/03. html (dernière consultation le 20 septembre 2019).

\section{MOE 2006a}

"Natsu no "uchi eko!" Aikon » no muryō daunrōdo kaishi ni tsuite『夏の「うち エコ!」アイコン』の無料ダウンロード開 始について (À propos de l'ouverture du téléchargement gratuit de "L'airconditionné “Uchi-eco!" pour l'été »), 2 avril.

http://www.env.go.jp/press/8237.html (dernière consultation le 20 septembre 2019).

\section{MOE 2006b}

Hon.nendo no « kūru bizu » no seika ni tsuite 本年度の「COOL BIZ」の成果につい $\tau$ (À propos des résultats de "Cool Biz » cette année), 10 novembre.

http://www.env.go.jp/press/press. php?serial=7690 (dernière consultation le 20 septembre 2019). 
MOE 2016

Heisei nijūkyū nendo kankyōshō jūten shisakushū 平成29年度環境省重点施策集 (Mesures prioritaires pour le budget de l'année fiscale 2017).

http://www.env.go.jp/guide/budget/ h29/h29juten-sesakushu.html (dernière consultation le 20 septembre 2019).

\section{MOE 2017}

Nihonban najji yunitto o hossoku shimasu 日本版ナッジ・ユニットを発足します (La version japonaise de la Nudge Unit est fondée), 14 avril.

https://www.env.go.jp/press/103926.html (dernière consultation le 20 septembre 2019).

NaKagamı Hidetoshi 中上英俊 2010 "Shōene no kagi wa "shōhisha" ni ari »省エネのカギは「消費者」にあり (Les « consommateurs » sont la clef des shōene), Nikkei shinbun 日経新聞, 28 décembre.

http://www.nikkei.com/article/ DGXNASDD2404X_U0A221C1000000/ (dernière consultation le 20 septembre 2019).

NishimuRA Kiyoshi 西村陽 2014 Nihon de no enerugì manejimento no genjō to tenbō 日本でのエネルギー・マ ネジメントの現状と展望 (Situation et perspectives sur la gestion de l'énergie au Japon), 18 février 2014 (transmis en main propre par l'auteur).

Nishioka Shūzō 西岡秀三 \& MoRitA Tsuneyuki 森田恒幸 1992 "Chikyū kikō anteika no tame no shakai keizai shisutemu no kōzō tenkan seisaku no taikei Kosumo puran II » 地球気候安 定化のための社会経済システムの構造 転換政策の体系〜 コスモプランII (Structurer des politiques de transformation des systèmes socioéconomiques afin de stabiliser le climat planétaire : COSMO Plan II), Kankyō kenkyū 環境研究, 86 : 6-13.

\section{Oshitanı Shizuka 2006}

Global Warming Policy in Japan and Britain. Interactions Between Institutions and Issue Characteristics, Manchester, Manchester University Press.

SAnPei Yuki \& Aoyagi-Usui Midori 2009 «Mass-media coverage, its influence on public awareness of climate change issues, and implications for Japan's national campaign to reduce greenhouse gas emissions ", Global Environmental Change, $19: 203-212$.

\section{SAWA Akihiro 澤昭裕 2010}

Eko bōkokuron エコ亡国論 (Théorie de l'effondrement de l'État causée par la politique écologique), Tokyo, Shinchōsha 新潮社.

\section{SCALise Paul J. 2013}

«Policy Images, Issue Frames, and Technical Realities: Contrasting Views of Japan's Energy Policy Development ", Social Science Japan Journal, 16 (2) : 301-308.

\section{SEEB 2014}

Setsuritsu shushi 設立趣旨 (Charte de la SEEB), Shōenerugī kōdō kenkyū-kai 省エネルギー行動研究会.

http://seeb.jp/about (dernière consultation le 20 septembre 2019). 
SLADE Toby 2009

Japanese Fashion. A Cultural History, Oxford, Berg Publishers.

\section{SHOve Elizabeth 2010}

«Beyond the ABC: Climate Change Policy and Theories of Social Change", Environment and Planning A, 42 (6) : 1273-1285.

SunikKa-Blank Minna \& Iwafune Yumiko 2011

« Sustainable Building in Japan -

Observations on a Market

Transformation Policy », Environmental

Policy and Governance, 21 : 351-363.

\section{Tan Chun Knee, Ogawa Akinori \&}

Matsumura Takashi 2008

Innovative Climate Change

Communication: Team Minus $6 \%$, GEIC

Working Paper Series 2008-001, United Nations University.

Thaler Richard H. \& Sunstein Cass R. 2008

Nudge. Improving Decisions about Health, Wealth, and Happiness, Yale, Yale University Press.

\section{Thomann Bernard 2010}

«Gouverner les ouvriers dans le Japon d'avant-guerre : la modernisation comme technique de gestion des populations », Ebisu. Études japonaises, 44 : 67-85.

\section{Thomann Bernard 2015}

La Naissance de l'État social japonais, Paris, Presses de Sciences Po.
Hiraga Kōichi 平賀功一 2015

"Sandome no "Jūtaku eko pointo" wa hontōni otoku ka »3度目の「住宅エコ ポイント」は本当にお得か (Le troisième « programme résidentiel écopoints » vaut-il vraiment le coup?), Tōyō Keizai 東洋経済, 8 février.

Walravens Tine 2017

«Food Safety and Regulatory Change Since the "Mad Cow" in Japan: Science, Self-Responsibility, and Trust ", Contemporary Japan, 29 (1) : 67-88.

Watanabe Rie 2011

Climate Policy Changes in Germany and Japan. A Path to Paradigmatic Policy Change, London, Routledge Research in Comparative Politics.

Westney Eleanor 1982

«The Emulation of Western

Organizations in Meiji Japan: The Case of the Paris Prefecture of Police and the Keishi-Cho », Journal of Japanese Studies, 8 (2) : 307-342.

Wilhite Harold, Nakagami Hidetoshi \& Masuda Takashi 1996

«A Cross Cultural Analysis of EnergyUse Behavior in Japan and Norway ", Energy Policy, 24 (9) : 795-803.

\section{YamaJ Kenji 2015}

« Japan's Energy and Environmental Strategy », in Kaya Yoichi, Yamaji Kenji \& Akimoto Keigo (dir.), Climate Change and Energy. Japanese Perspectives on Climate Change Mitigation Strategies, Londres, Imperial College Press : 57-132. 